\title{
A Comprehensive Targeted Metabolomic Assay for Urine Analysis
}

Jiamin Zheng ${ }^{1}$, Lun Zhang ${ }^{1}$, Mathew Johnson ${ }^{1}$, Rupasri Mandal ${ }^{1},{ }^{*}$ David S. Wishart $^{1,2}$

Departments of Biological Sciences ${ }^{1}$ and Computing Science ${ }^{2}$, University of Alberta, Edmonton, AB, Canada T6G 2R3

*Author to whom correspondence should be addressed: Dr. David S. Wishart E-Mail: dwishart@ualberta.ca 


\section{Table of Contents}

Supplementary Document 1. Detailed chemical vendor information .................................................... 1

Table S1. Concentration of Standards at Different Calibration Levels....................................................S3

Table S2. Optimized MRM Transitions and Mass Spectrometer Parameters........................................... S5

Table S3. Calibration Regression, LOD, LLOQ and ULOQ ............................................................... S9

Table S4. Intra- and Inter-day Accuracy and Precision ....................................................................... S11

Table S5. Recovery Performance in Spiked Pooled Human Urine ..................................................... S14

Table S6. Validation Performance of Analytes Analyzed via DFI-MS/MS ...........................................S16

Table S7. Method Comparison to Other Urinary Assays ........................................................................ S19 


\section{Supplementary Document 1. Detailed chemical vendor information}

Chemical standards including acetylornithine, asymmetric dimethylarginine (ADMA), carnosine, creatinine, levodopa (DOPA), dopamine, histamine, methionine sulfoxide, cis-hydroxyproline, trans-hydroxyproline, phenylethylamine, putrescine, sarcosine, serotonin, spermidine, spermine, taurine, tyramine, alanine, arginine, asparagine, aspartic acid, citrulline, glutamine, glutamic acid, glycine, histidine, leucine, isoleucine, lysine, methionine, ornithine, phenylalanine, proline, serine, threonine, tryptophan, valine, 3-methylhistidine, betaine, trimethylamine $\mathrm{N}$-oxide (TMAO), choline, nitro-tyrosine, tyrosine, kynurenine, creatine, alpha-aminoadipic acid, lactic acid, betahydroxybutyric acid, alpha-ketoglutaric acid, citric acid, butyric acid, isobutyric acid, propionic acid, succinic acid, fumaric acid, pyruvic acid, hippuric acid, methylmalonic acid, homovanillic acid, indole-3-acetic acid, uric acid, L-carnitine inner salt, acetyl-L-carnitine hydrochloride, propionyl-L-carnitine, butyryl-L-carnitine, hexanoyl-L-carnitine, octanoyl-L-carnitine, decanoylL-carnitine, dodecanoyl-L-carnitine, tetradecanoyl-L-carnitine, hexadecanoyl-L-carnitine, octadecanoyl-L-carnitine, N-stearoyl-D-erythro-sphingosylphosphorylcholine, 1,2-dilinolenoylsn-glycero-3-phosphocholine, 1,2-Dioctadecanoyl-sn-glycero-3-phosphocholine, 1-oleoyl-2hydroxy-sn-glycero-3-phosphocholine, and glucose were all bought from Sigma-Aldrich (Oakville, ON, CA). $\mathrm{N}^{1}, \mathrm{~N}^{12}$-diacetylspermine hydrochloride was purchased from Cayman Chemical (Ann Arbor, MI, U.S.A.). 4-hydroxyhippuric acid was obtained from Toronto Research Chemicals (North York, ON, CA). Other stable isotope (D, $\left.{ }^{13} \mathrm{C},{ }^{15} \mathrm{~N}\right)$-labelled internal standards (ISTD) including $\mathrm{D}_{2}$-ornithine, ${ }^{15} \mathrm{~N}$-histidine, $\mathrm{D}_{3}$-creatinine, $\mathrm{D}_{3}$-DOPA, $\mathrm{D}_{4}$-dopamine, ${ }^{13} \mathrm{C}$ tyrosine, ${ }^{13} \mathrm{C}$ - $\mathrm{D}_{3}$-methionine, $\mathrm{D}_{3}$-proline, $\mathrm{D}_{4}$-serotonin, $\mathrm{D}_{4}$-putrescine, $\mathrm{D}_{3}$-sarcosine, ${ }^{13} \mathrm{C}_{2}$-taurine, $\mathrm{D}_{4}$-tyramine, ${ }^{15} \mathrm{~N}$-alanine, ${ }^{13} \mathrm{C}_{6}$-arginine, ${ }^{15} \mathrm{~N}$-asparagine, $\mathrm{D}_{3}$-aspartic acid, $\mathrm{D}_{7}$-citrulline, $\mathrm{D}_{3}$ glutamic acid, $\mathrm{D}_{5}$-glutamine, ${ }^{13} \mathrm{C}_{2}$-glycine, ${ }^{13} \mathrm{C}$-leucine, ${ }^{15} \mathrm{~N}$-phenylalanine, ${ }^{13} \mathrm{C}$-serine, $\mathrm{D}_{2}$ threonine, ${ }^{15} \mathrm{~N}_{2}$-tryptophan, $\mathrm{D}_{8}$-valine, $\mathrm{D}_{9}$-TMAO and ${ }^{15} \mathrm{~N}_{2}$-uric acid were bought from Cambridge 
Isotope Laboratories Inc. (Tewksbury, MA, U.S.A.). D8-spermine tetrahydrochloride, $\mathrm{D}_{8}$ spermidine trihydrochloride were purchased from IsoSciences (Ambler, PA, U.S.A.). $\mathrm{D}_{6}-\mathrm{N}^{1}, \mathrm{~N}^{12}$ diacetylspermine dihydrochloride and $\mathrm{D}_{6}$-ADMA were bought from Toronto Research Chemicals (North York, ON, CA). D3-creatinine, D9-choline chloride, D9-betaine hydrochloride, 2-D ${ }_{1}$ sodium L-lactate, $\mathrm{D}_{4}$-sodium beta-hydroxybutyrate, $1-{ }^{13} \mathrm{C}$-alpha-ketoglutaric acid, $\mathrm{D}_{4}$-citric acid, $1-{ }^{13} \mathrm{C}$-butyric acid, $1-{ }^{13} \mathrm{C}$-propionic acid, $\mathrm{D}_{4}$-succinic acid, ${ }^{13} \mathrm{C}_{2}-\mathrm{D}_{2}$-fumaric acid, ${ }^{13} \mathrm{C}$-pyruvic acid, $\mathrm{D}_{2}$-hippuric acid, methyl- $\mathrm{D}_{3}$-malonic acid, and $\mathrm{D}_{2}$-indole-3-acetic acid were purchased from C/D/N Isotopes Inc. (Pointe-Claire, QC, CA). Labelled carnitine standard set was purchased from Cambridge Isotope Laboratories Inc. (Tewksbury, MA, U.S.A.). 1,2-dimyristoyl-sn-glycero-3phosphocholine, 1,2-diarachidoyl-sn-glycero-3-phosphocholine, 1-nonanoyl-2-hydroxy-snglycero-3-phosphocholine, N-hexanoyl-D-erythro-sphingosylphosphorylcholine, and ${ }^{13} \mathrm{C}_{6}$ glucose were bought from Sigma-Aldrich (Oakville, ON, CA). 
Table S1. Concentration of Standards at Different Calibration Levels

\begin{tabular}{|c|c|c|c|c|c|c|c|}
\hline \multirow{2}{*}{ Analyte } & \multicolumn{7}{|c|}{ Concentration in $\mu \mathrm{M}$} \\
\hline & Cal1 & Cal2 & Cal3 & Cal4 & Cal5 & Cal6 & Cal7 \\
\hline Creatinine & 300 & 600 & 3000 & 6000 & 12000 & 18000 & 24000 \\
\hline Glycine & 25 & 50 & 250 & 500 & 1000 & 1500 & 2000 \\
\hline Alanine & 20 & 40 & 200 & 400 & 800 & 1200 & 1600 \\
\hline Serine & 15 & 30 & 150 & 300 & 600 & 900 & 1200 \\
\hline Histamine & 0.1 & 0.2 & 1 & 2 & 4 & 6 & 8 \\
\hline Proline & 2 & 4 & 20 & 40 & 80 & 120 & 160 \\
\hline Valine & 2.5 & 5 & 25 & 50 & 100 & 150 & 200 \\
\hline Threonine & 5 & 10 & 50 & 100 & 200 & 300 & 400 \\
\hline Phenylethylamine & 0.1 & 0.2 & 1 & 2 & 4 & 6 & 8 \\
\hline Taurine & 20 & 40 & 200 & 400 & 800 & 1200 & 1600 \\
\hline Putrescine & 0.5 & 1 & 5 & 10 & 20 & 30 & 40 \\
\hline cis-Hydroxyproline & 0.5 & 1 & 5 & 10 & 20 & 30 & 40 \\
\hline trans-Hydroxyproline & 0.5 & 1 & 5 & 10 & 20 & 30 & 40 \\
\hline Leucine & 1 & 2 & 10 & 20 & 40 & 60 & 80 \\
\hline Isoleucine & 0.5 & 1 & 5 & 10 & 20 & 30 & 40 \\
\hline Asparagine & 5 & 10 & 50 & 100 & 200 & 300 & 400 \\
\hline Aspartic acid & 5 & 10 & 50 & 100 & 200 & 300 & 400 \\
\hline Glutamine & 20 & 40 & 200 & 400 & 800 & 1200 & 1600 \\
\hline Glutamic acid & 5 & 10 & 50 & 100 & 200 & 300 & 400 \\
\hline Methionine & 20 & 40 & 200 & 400 & 800 & 1200 & 1600 \\
\hline Dopamine & 0.5 & 1 & 5 & 10 & 20 & 30 & 40 \\
\hline Histidine & 25 & 50 & 250 & 500 & 1000 & 1500 & 2000 \\
\hline alpha-aminoadipic acid & 2.5 & 5 & 25 & 50 & 100 & 150 & 200 \\
\hline Phenylalanine & 5 & 10 & 50 & 100 & 200 & 300 & 400 \\
\hline Methionine-Sulfoxide & 1 & 2 & 10 & 20 & 40 & 60 & 80 \\
\hline Arginine & 2.5 & 5 & 25 & 50 & 100 & 150 & 200 \\
\hline Acetyl-ornithine & 0.5 & 1 & 5 & 10 & 20 & 30 & 40 \\
\hline Citrulline & 1.25 & 2.5 & 12.5 & 25 & 50 & 75 & 100 \\
\hline Serotonin & 0.1 & 0.2 & 1 & 2 & 4 & 6 & 8 \\
\hline Tyrosine & 5 & 10 & 50 & 100 & 200 & 300 & 400 \\
\hline DOPA & 0.5 & 1 & 5 & 10 & 20 & 30 & 40 \\
\hline Asymmetric dimethylarginine & 0.5 & 1 & 5 & 10 & 20 & 30 & 40 \\
\hline Total dimethylarginine & 0.5 & 1 & 5 & 10 & 20 & 30 & 40 \\
\hline Tryptophan & 5 & 10 & 50 & 100 & 200 & 300 & 400 \\
\hline Kynurenine & 1 & 2 & 10 & 20 & 40 & 60 & 80 \\
\hline Carnosine & 2 & 4 & 20 & 40 & 80 & 120 & 160 \\
\hline Nitro-Tyrosine & 1 & 2 & 10 & 20 & 40 & 60 & 80 \\
\hline
\end{tabular}




\begin{tabular}{|c|c|c|c|c|c|c|c|}
\hline Ornithine & 5 & 10 & 50 & 100 & 200 & 300 & 400 \\
\hline Lysine & 10 & 20 & 100 & 200 & 400 & 600 & 800 \\
\hline Spermidine & 0.25 & 0.5 & 2.5 & 5 & 10 & 15 & 20 \\
\hline Spermine & 0.25 & 0.5 & 2.5 & 5 & 10 & 15 & 20 \\
\hline Sarcosine & 0.5 & 1 & 5 & 10 & 20 & 30 & 40 \\
\hline Diacetylspermine & 0.25 & 0.5 & 2.5 & 5 & 10 & 15 & 20 \\
\hline Tyramine & 0.5 & 1 & 5 & 10 & 20 & 30 & 40 \\
\hline Creatine & 25 & 50 & 250 & 500 & 1000 & 1500 & 2000 \\
\hline Phosphocreatine & 0.25 & 0.5 & 2.5 & 5 & 10 & 15 & 20 \\
\hline Betaine & 5 & 10 & 50 & 100 & 200 & 300 & 400 \\
\hline Choline & 2 & 4 & 20 & 40 & 80 & 120 & 160 \\
\hline TMAO & 50 & 100 & 500 & 1000 & 2000 & 3000 & 4000 \\
\hline Methylhistidine & 10 & 20 & 100 & 200 & 400 & 600 & 800 \\
\hline Lactic acid & 12.5 & 25 & 50 & 100 & 200 & 400 & 1000 \\
\hline Beta-Hydroxybutyric acid & 2.5 & 5 & 10 & 20 & 40 & 80 & 200 \\
\hline Alpha-Ketoglutaric acid & 5 & 10 & 20 & 40 & 80 & 160 & 400 \\
\hline Citric acid & 100 & 200 & 400 & 800 & 1600 & 3200 & 8000 \\
\hline Butyric acid & 1 & 2 & 4 & 8 & 16 & 32 & 80 \\
\hline Propionic acid & 1 & 2 & 4 & 8 & 16 & 32 & 80 \\
\hline HPHPA & 5 & 10 & 20 & 40 & 80 & 160 & 400 \\
\hline para-Hydroxyhippuric acid & 2.5 & 5 & 10 & 20 & 40 & 80 & 200 \\
\hline Succinic acid & 5 & 10 & 20 & 40 & 80 & 160 & 400 \\
\hline Fumaric acid & 0.5 & 1 & 2 & 4 & 8 & 16 & 40 \\
\hline Pyruvic acid & 2.5 & 5 & 10 & 20 & 40 & 80 & 200 \\
\hline Isobutyric acid & 2.5 & 5 & 10 & 20 & 40 & 80 & 200 \\
\hline Hippuric acid & 50 & 100 & 200 & 400 & 800 & 1600 & 4000 \\
\hline Methylmalonic acid & 1 & 2 & 4 & 8 & 16 & 32 & 80 \\
\hline Homovanillic acid & 2.5 & 5 & 10 & 20 & 40 & 80 & 200 \\
\hline Indole acetic acid & 2.5 & 5 & 10 & 20 & 40 & 80 & 200 \\
\hline Uric acid & 50 & 100 & 200 & 400 & 800 & 1600 & 4000 \\
\hline
\end{tabular}


Table S2. Optimized MRM Transitions and Mass Spectrometer Parameters

\begin{tabular}{|c|c|c|c|c|c|}
\hline \multirow{2}{*}{ Analyte } & \multicolumn{5}{|c|}{ MRM Optimization } \\
\hline & Q1 & Q3 & DP & CE & CXP \\
\hline Creatinine & 114.1 & 44.1 & 60 & 17 & 15 \\
\hline Glycine & 211.2 & 75.9 & 66 & 14 & 15 \\
\hline Alanine & 225.2 & 44.2 & 61 & 35 & 15 \\
\hline Serine & 241.2 & 60 & 41 & 31 & 15 \\
\hline Histamine & 247 & 154 & 56 & 19 & 15 \\
\hline Proline & 251.2 & 70.3 & 51 & 43 & 15 \\
\hline Valine & 253.2 & 72.2 & 81 & 31 & 15 \\
\hline Threonine & 255.2 & 74.1 & 66 & 29 & 15 \\
\hline Phenylethylamine & 257.2 & 105.2 & 71 & 31 & 15 \\
\hline Taurine & 261 & 126.1 & 71 & 21 & 15 \\
\hline Putrescine & 266.1 & 113.9 & 100 & 25 & 15 \\
\hline cis-Hydroxyproline & 267.1 & 68 & 56 & 61 & 15 \\
\hline trans-Hydroxyproline & 267.15 & 68 & 56 & 61 & 15 \\
\hline Leucine & 267.3 & 43 & 55 & 66 & 15 \\
\hline Isoleucine & 267.3 & 69 & 55 & 45 & 15 \\
\hline Asparagine & 268.2 & 87 & 41 & 25 & 15 \\
\hline Aspartic acid & 269.2 & 116.2 & 76 & 21 & 15 \\
\hline Glutamine & 282.2 & 130 & 71 & 25 & 15 \\
\hline Glutamic acid & 283.2 & 130.2 & 76 & 23 & 15 \\
\hline Methionine & 285.1 & 104.2 & 71 & 25 & 15 \\
\hline Dopamine & 289.2 & 137.2 & 56 & 29 & 15 \\
\hline Histidine & 291.1 & 110.2 & 56 & 33 & 15 \\
\hline alpha-aminoadipic acid & 297.1 & 144.2 & 46 & 23 & 15 \\
\hline Phenylalanine & 301.2 & 120.2 & 71 & 31 & 15 \\
\hline Methionine-Sulfoxide & 301.2 & 88.1 & 61 & 41 & 15 \\
\hline Arginine & 310 & 217 & 76 & 23 & 15 \\
\hline Acetyl-ornithine & 310.2 & 217.3 & 46 & 15 & 15 \\
\hline Citrulline & 311.2 & 113.1 & 56 & 29 & 15 \\
\hline Serotonin & 312.3 & 160.2 & 66 & 27 & 15 \\
\hline Tyrosine & 317.2 & 136.1 & 76 & 31 & 15 \\
\hline DOPA & 333.1 & 198.1 & 61 & 19 & 15 \\
\hline Asymmetric dimethylarginine & 338.2 & 46 & 71 & 61 & 15 \\
\hline Total dimethylarginine & 338.2 & 70.1 & 71 & 61 & 15 \\
\hline Tryptophan & 340.2 & 188.2 & 96 & 25 & 15 \\
\hline Kynurenine & 344.2 & 146.2 & 46 & 33 & 15 \\
\hline Carnosine & 362.2 & 110.1 & 46 & 47 & 15 \\
\hline Nitro-Tyrosine & 362.2 & 136.1 & 65 & 41 & 15 \\
\hline
\end{tabular}




\begin{tabular}{|c|c|c|c|c|c|}
\hline Ornithine & 403.2 & 310.2 & 76 & 19 & 15 \\
\hline Lysine & 417.2 & 324.2 & 81 & 19 & 15 \\
\hline Spermidine & 551.2 & 193.2 & 71 & 41 & 15 \\
\hline Spermine & 743.3 & 193.2 & 56 & 61 & 15 \\
\hline Sarcosine & 225.1 & 44.2 & 43 & 31 & 15 \\
\hline Diacetylspermine & 422.4 & 171.2 & 32 & 34 & 15 \\
\hline Tyramine & 273.2 & 121.1 & 41 & 29 & 15 \\
\hline Creatine & 132.1 & 90 & 40 & 18 & 15 \\
\hline Phosphocreatine & 212.1 & 90.1 & 40 & 21 & 15 \\
\hline Betaine & 118.1 & 59.2 & 60 & 30 & 15 \\
\hline Choline & 104.2 & 60.1 & 28 & 50 & 15 \\
\hline TMAO & 76 & 58 & 40 & 26 & 15 \\
\hline Methylhistidine & 305.1 & 212.1 & 46 & 23 & 15 \\
\hline Lactic acid & 224 & 137 & -55 & -12 & -10 \\
\hline Beta-Hydroxybutyric acid & 238 & 193.8 & -58 & -21 & -6 \\
\hline Alpha-Ketoglutaric acid & 550.1 & 232.7 & -104 & -36 & -24 \\
\hline Citric acid & 596.3 & 221.9 & -92 & -50 & -16 \\
\hline Butyric acid & 221.9 & 136.8 & -68 & -30 & -5 \\
\hline Propionic acid & 208 & 136.8 & -30 & -26 & -25 \\
\hline HPHPA & 316.2 & 194.2 & -70 & -20 & -9 \\
\hline para-Hydroxyhippuric acid & 328.9 & 208.8 & -91 & -28 & -9 \\
\hline Succinic acid & 387.1 & 234 & -100 & -27 & -20 \\
\hline Fumaric acid & 385 & 234 & -78 & -20 & -9 \\
\hline Pyruvic acid & 357 & 136.8 & -80 & -33 & -5 \\
\hline Isobutyric acid & 221.9 & 136.7 & -80 & -28 & -9 \\
\hline Hippuric acid & 313 & 136.9 & -65 & -26 & -11 \\
\hline Methylmalonic acid & 387 & 177.9 & -85 & -28 & -9 \\
\hline Homovanillic acid & 316 & 149.8 & -79 & -26 & -10 \\
\hline Indole acetic acid & 308.9 & 115.9 & -100 & -28 & -16 \\
\hline Uric acid & 167.1 & 124.1 & -40 & -20 & -6 \\
\hline Hexose & 179 & 89 & -55 & -12 & -15 \\
\hline LysoPC a C14:0 & 468.3 & 184 & 79 & 30 & 15 \\
\hline LysoPC a C16:1 & 494.3 & 184 & 79 & 30 & 15 \\
\hline LysoPC a C16:0 & 496.3 & 184 & 81 & 31 & 15 \\
\hline LysoPC a C17:0 & 510.3 & 184 & 82 & 31 & 15 \\
\hline LysoPC a C18:2 & 520.3 & 184 & 82 & 31 & 15 \\
\hline LysoPC a C18:1 & 522.3 & 184 & 82 & 31 & 15 \\
\hline LysoPC a C18:0 & 524.3 & 184 & 85 & 31 & 15 \\
\hline LysoPC a C20:4 & 544.3 & 184 & 85 & 31 & 15 \\
\hline LysoPC a C20:3 & 546.3 & 184 & 87 & 31 & 15 \\
\hline LysoPC a C24:0 & 608.4 & 184 & 96 & 33 & 15 \\
\hline LysoPC a C26:1 & 634.4 & 184 & 98 & 33 & 15 \\
\hline LysoPC a C26:0 & 636.5 & 184 & 101 & 34 & 15 \\
\hline
\end{tabular}




\begin{tabular}{|c|c|c|c|c|c|}
\hline LysoPC a C28:1 & 662.5 & 184 & 125 & 53 & 15 \\
\hline LysoPC a C28:0 & 664.5 & 184 & 105 & 35 & 15 \\
\hline $\mathrm{SM}(\mathrm{OH}) \mathrm{C} 14: 1$ & 689.6 & 184 & 108 & 36 & 15 \\
\hline SM C16:1 & 701.6 & 184 & 111 & 37 & 15 \\
\hline SM C16:0 & 703.6 & 184 & 112 & 37 & 15 \\
\hline $\mathrm{SM}(\mathrm{OH}) \mathrm{C} 16: 1$ & 717.6 & 184 & 114 & 38 & 15 \\
\hline SM C18:1 & 729.6 & 184 & 117 & 38 & 15 \\
\hline PC aa C32:2 & 730.5 & 184 & 117 & 38 & 15 \\
\hline SM C18:0 & 731.6 & 184 & 117 & 38 & 15 \\
\hline SM C20:2 & 755.6 & 184 & 123 & 40 & 15 \\
\hline PC ae C36:0 & 776.7 & 184 & 128 & 42 & 15 \\
\hline PC aa C36:6 & 778.5 & 184 & 128 & 42 & 15 \\
\hline PC aa C36:0 & 790.6 & 184 & 131 & 43 & 15 \\
\hline $\mathrm{SM}(\mathrm{OH}) \mathrm{C} 22: 2$ & 799.7 & 184 & 133 & 43 & 15 \\
\hline $\mathrm{SM}(\mathrm{OH}) \mathrm{C} 22: 1$ & 801.7 & 184 & 134 & 43 & 15 \\
\hline PC aa C38:6 & 806.6 & 184 & 135 & 44 & 15 \\
\hline Pc aa C38:0 & 818.7 & 184 & 138 & 45 & 15 \\
\hline Pc ae $\mathrm{C} 40: 6$ & 820.6 & 184 & 139 & 45 & 15 \\
\hline $\mathrm{SM}(\mathrm{OH}) \mathrm{C} 24: 1$ & 829.7 & 184 & 141 & 46 & 15 \\
\hline PC aa C40:6 & 834.6 & 184 & 143 & 46 & 15 \\
\hline PC aa C40:2 & 842.7 & 184 & 144 & 47 & 15 \\
\hline PC aa C40:1 & 844.7 & 184 & 145 & 47 & 15 \\
\hline $\mathrm{CO}$ & 162.1 & 85.1 & 61 & 27 & 15 \\
\hline $\mathrm{C} 2$ & 204.1 & 85.1 & 41 & 27 & 15 \\
\hline C3:1 & 216.1 & 85.1 & 49 & 27 & 15 \\
\hline $\mathrm{C} 3$ & 218.1 & 85.1 & 46 & 29 & 15 \\
\hline$C 4: 1$ & 230.1 & 85.1 & 52 & 29 & 15 \\
\hline $\mathrm{C} 4$ & 232.2 & 85.1 & 46 & 29 & 15 \\
\hline $\mathrm{C} 3 \mathrm{OH}$ & 234.1 & 85.1 & 53 & 30 & 15 \\
\hline C5:1 & 244.2 & 85.1 & 55 & 31 & 15 \\
\hline $\mathrm{C} 5$ & 246.2 & 85.1 & 46 & 29 & 15 \\
\hline $\mathrm{C} 4 \mathrm{OH}$ & 248.1 & 85.1 & 55 & 32 & 15 \\
\hline C6:1 & 258.1 & 85.1 & 57 & 33 & 15 \\
\hline C6 & 260.2 & 85.1 & 56 & 27 & 15 \\
\hline $\mathrm{C5OH}$ & 262.2 & 85.1 & 58 & 33 & 15 \\
\hline C5:1DC & 274.1 & 85.1 & 60 & 35 & 15 \\
\hline C5DC & 276.2 & 85.1 & 61 & 35 & 15 \\
\hline $\mathrm{C} 8$ & 288.2 & 85.1 & 66 & 33 & 15 \\
\hline C5MDC & 290.2 & 85.1 & 63 & 37 & 15 \\
\hline C9 & 302.2 & 85.1 & 66 & 39 & 15 \\
\hline C7DC & 304.2 & 85.1 & 66 & 39 & 15 \\
\hline C10:2 & 312.2 & 85.1 & 67 & 40 & 15 \\
\hline C10:1 & 314.2 & 85.1 & 68 & 40 & 15 \\
\hline
\end{tabular}




\begin{tabular}{|c|c|c|c|c|c|}
\hline $\mathrm{C} 10$ & 316.2 & 85.1 & 56 & 37 & 15 \\
\hline $\mathrm{C} 12: 1$ & 342.2 & 85.1 & 73 & 44 & 15 \\
\hline $\mathrm{C} 12$ & 344.3 & 85.1 & 73 & 44 & 15 \\
\hline $\mathrm{C} 14: 2$ & 368.3 & 85.1 & 78 & 47 & 15 \\
\hline $\mathrm{C} 14: 1$ & 370.3 & 85.1 & 78 & 47 & 15 \\
\hline $\mathrm{C} 14$ & 372.2 & 85.1 & 86 & 45 & 15 \\
\hline $\mathrm{C} 12 \mathrm{DC}$ & 374.3 & 85.1 & 86 & 45 & 15 \\
\hline $\mathrm{C} 14: 2 \mathrm{OH}$ & 384.3 & 85.1 & 81 & 49 & 15 \\
\hline $\mathrm{C} 14: 1 \mathrm{OH}$ & 386.3 & 85.1 & 81 & 50 & 15 \\
\hline $\mathrm{C} 16: 2$ & 396.3 & 85.1 & 83 & 51 & 15 \\
\hline $\mathrm{C} 16: 1$ & 398.3 & 85.1 & 84 & 51 & 15 \\
\hline $\mathrm{C} 16$ & 400.3 & 85.1 & 84 & 51 & 15 \\
\hline $\mathrm{C} 16: 2 \mathrm{OH}$ & 412.3 & 85.1 & 86 & 53 & 15 \\
\hline $\mathrm{C} 16: 10 \mathrm{H}$ & 414.3 & 85.1 & 87 & 53 & 15 \\
\hline $\mathrm{C} 16 \mathrm{OH}$ & 416.3 & 85.1 & 87 & 53 & 15 \\
\hline $\mathrm{C} 18: 2$ & 424.3 & 85.1 & 89 & 54 & 15 \\
\hline $\mathrm{C} 18: 1$ & 426.4 & 85.1 & 89 & 55 & 15 \\
\hline $\mathrm{C} 18$ & 428.4 & 85.1 & 96 & 63 & 15 \\
\hline $\mathrm{C} 18: 10 \mathrm{H}$ & 442.4 & 85.1 & 92 & 57 & 15 \\
\hline
\end{tabular}


Table S3. Calibration Regression, LOD, LLOQ and ULOQ

\begin{tabular}{|c|c|c|c|c|}
\hline Analyte & $\begin{array}{c}\text { Correlation Coefficient } \\
\left(\mathbf{R}^{2}\right)\end{array}$ & LOD $(\mu \mathrm{M})$ & LLOQ ( $\mu \mathrm{M})$ & ULOQ $(\mu \mathrm{M})$ \\
\hline Creatinine & 0.9998 & 1.57 & 300 & 24000 \\
\hline Glycine & 0.9994 & 0.299 & 25 & 2000 \\
\hline Alanine & 0.9986 & 0.699 & 20 & 1600 \\
\hline Serine & 0.9994 & 0.137 & 15 & 1200 \\
\hline Histamine & 0.9962 & 0.00324 & 0.1 & 8 \\
\hline Proline & 0.9998 & 0.0627 & 2 & 160 \\
\hline Valine & 0.9984 & 0.0885 & 2.5 & 200 \\
\hline Threonine & 0.9996 & 0.315 & 5 & 400 \\
\hline Phenylethylamine & 0.9980 & 0.00319 & 0.1 & 8 \\
\hline Taurine & 0.9976 & 0.446 & 20 & 1600 \\
\hline Putrescine & 0.9986 & 0.00673 & 0.5 & 40 \\
\hline cis-Hydroxyproline & 0.9994 & 0.0492 & 0.5 & 40 \\
\hline trans-Hydroxyproline & 0.9978 & 0.0244 & 0.5 & 40 \\
\hline Leucine & 0.9978 & 0.545 & 1 & 80 \\
\hline Isoleucine & 0.9992 & 0.200 & 0.5 & 40 \\
\hline Asparagine & 0.9994 & 0.325 & 5 & 400 \\
\hline Aspartic acid & 0.9970 & 2.73 & 5.00 & 400 \\
\hline Glutamine & 0.9992 & 0.185 & 20 & 1600 \\
\hline Glutamic acid & 0.9966 & 0.698 & 5 & 400 \\
\hline Methionine & 0.9996 & 0.165 & 20 & 1600 \\
\hline Dopamine & 0.9984 & 0.00442 & 0.5 & 40 \\
\hline Histidine & 0.9994 & 0.0353 & 25 & 2000 \\
\hline alpha-aminoadipic acid & 0.9994 & 0.161 & 2.5 & 200 \\
\hline Phenylalanine & 0.9976 & 0.0207 & 5 & 400 \\
\hline Methionine-Sulfoxide & 0.9974 & 0.0923 & 1 & 80 \\
\hline Arginine & 0.9956 & 0.0743 & 2.5 & 200 \\
\hline Acetyl-ornithine & 0.9982 & 0.0265 & 0.5 & 40 \\
\hline Citrulline & 0.9992 & 0.197 & 1.25 & 100 \\
\hline Serotonin & 0.9992 & 0.00594 & 0.1 & 8 \\
\hline Tyrosine & 0.9968 & 0.0726 & 5 & 400 \\
\hline DOPA & 0.9956 & 0.0112 & 0.5 & 40 \\
\hline Asymmetric dimethylarginine & 0.9994 & 0.0167 & 0.5 & 40 \\
\hline Total dimethylarginine & 0.9996 & 0.0103 & 0.5 & 40 \\
\hline Tryptophan & 0.9994 & 0.0186 & 5 & 400 \\
\hline Kynurenine & 0.9962 & 0.0754 & 1 & 80 \\
\hline Carnosine & 0.9988 & 0.0271 & 2 & 160 \\
\hline Nitro-Tyrosine & 0.9994 & 0.0517 & 1 & 80 \\
\hline Ornithine & 0.9992 & 0.0441 & 5 & 400 \\
\hline Lysine & 0.9988 & 0.105 & 10 & 800 \\
\hline
\end{tabular}




\begin{tabular}{|c|c|c|c|c|}
\hline Spermidine & 0.9998 & 0.00231 & 0.25 & 20 \\
\hline Spermine & 0.9994 & 0.00537 & 0.25 & 20 \\
\hline Sarcosine & 0.9994 & 0.395 & 0.5 & 40 \\
\hline Diacetylspermine & 0.999 & 0.0395 & 0.25 & 20 \\
\hline Tyramine & 0.9986 & 0.0215 & 0.5 & 40 \\
\hline Creatine & 1.0000 & 0.455 & 25 & 2000 \\
\hline Phosphocreatine & 0.9976 & 0.136 & 0.25 & 20 \\
\hline Betaine & 0.9988 & 2.24 & 5 & 400 \\
\hline Choline & 0.9998 & 0.324 & 2 & 160 \\
\hline TMAO & 0.9998 & 0.408 & 50.00 & 4000 \\
\hline Methylhistidine & 0.9992 & 0.0251 & 10.000 & 800 \\
\hline Lactic acid & 0.9994 & 0.677 & 12.5 & 1000 \\
\hline Beta-Hydroxybutyric acid & 0.9996 & 0.174 & 2.5 & 200 \\
\hline Alpha-Ketoglutaric acid & 0.9988 & 0.241 & 5.000 & 400 \\
\hline Citric acid & 0.9962 & 2.92 & 100 & 8000 \\
\hline Butyric acid & 0.9980 & 0.0687 & 1 & 80 \\
\hline Propionic acid & 0.9970 & 0.0429 & 1 & 80 \\
\hline HPHPA & 0.9898 & 0.113 & 5 & 400 \\
\hline para-Hydroxyhippuric acid & 0.9980 & 0.268 & 2.5 & 200 \\
\hline Succinic acid & 0.9960 & 0.0411 & 5 & 400 \\
\hline Fumaric acid & 0.9912 & 0.0419 & 0.5 & 40 \\
\hline Pyruvic acid & 0.9982 & 0.0491 & 2.5 & 200 \\
\hline Isobutyric acid & 0.9984 & 0.0299 & 2.5 & 200 \\
\hline Hippuric acid & 0.9982 & 0.231 & 50 & 4000 \\
\hline Methylmalonic acid & 0.9998 & 0.0489 & 1 & 80 \\
\hline Homovanillic acid & 0.9986 & 0.369 & 2.5 & 200 \\
\hline Indole acetic acid & 0.9992 & 0.238 & 2.5 & 200 \\
\hline Uric acid & 0.9990 & 2.74 & 50 & 4000 \\
\hline
\end{tabular}


Table S4. Intra- and Inter-day Accuracy and Precision

\begin{tabular}{|c|c|c|c|c|c|c|c|c|c|c|c|c|c|c|c|c|}
\hline \multirow{3}{*}{ Analyte } & \multicolumn{4}{|c|}{ Lower } & \multicolumn{4}{|c|}{ Low } & \multicolumn{4}{|c|}{ Mid } & \multicolumn{4}{|c|}{ High } \\
\hline & \multicolumn{2}{|c|}{ Intra-day } & \multicolumn{2}{|c|}{ Inter-day } & \multicolumn{2}{|c|}{ Intra-day } & \multicolumn{2}{|c|}{ Inter-day } & \multicolumn{2}{|c|}{ Intra-day } & \multicolumn{2}{|c|}{ Inter-day } & \multicolumn{2}{|c|}{ Intra-day } & \multicolumn{2}{|c|}{ Inter-day } \\
\hline & $\begin{array}{c}\text { Accurac } \\
\text { y (\%) }\end{array}$ & CV (\%) & $\begin{array}{c}\text { Accurac } \\
\text { y (\%) }\end{array}$ & CV (\%) & $\begin{array}{c}\text { Accurac } \\
\text { y (\%) }\end{array}$ & CV (\%) & $\begin{array}{c}\text { Accurac } \\
\text { y (\%) }\end{array}$ & CV (\%) & $\begin{array}{c}\text { Accurac } \\
\text { y (\%) }\end{array}$ & CV (\%) & $\begin{array}{c}\text { Accurac } \\
\text { y (\%) }\end{array}$ & CV (\%) & $\begin{array}{c}\text { Accurac } \\
\text { y (\%) }\end{array}$ & CV (\%) & $\begin{array}{c}\text { Accurac } \\
\text { y (\%) }\end{array}$ & CV (\%) \\
\hline Creatinine & 97.8 & 1.31 & 96.8 & 2.59 & 102 & 2.16 & 106 & 3.48 & 97.9 & 1.03 & 102 & 3.58 & 100 & 1.08 & 102 & 5.33 \\
\hline Glycine & 105 & 4.87 & 104 & 4.72 & 98 & 2.89 & 107 & 7.39 & 97.2 & 2.45 & 107 & 4.92 & 106 & 3.23 & 102 & 4.81 \\
\hline Alanine & 111 & 2.19 & 102 & 7.17 & 111 & 2.94 & 109 & 5.16 & 102 & 2.28 & 104 & 3.82 & 97.1 & 3.80 & 103 & 8.00 \\
\hline Serine & 109 & 5.53 & 105 & 7.17 & 113 & 0.735 & 108 & 6.48 & 104 & 4.60 & 96.5 & 6.68 & 97.4 & 1.61 & 95.2 & 6.37 \\
\hline Histamine & 111 & 2.78 & 98.8 & 12.8 & 101 & 3.75 & 118 & 7.61 & 98.8 & 6.06 & 104 & 5.92 & 98.4 & 3.49 & 96.5 & 5.57 \\
\hline Proline & 99.5 & 1.73 & 101 & 6.88 & 102 & 0.353 & 99.3 & 4.51 & 101 & 1.26 & 95.8 & 4.98 & 97.4 & 0.926 & 100 & 4.21 \\
\hline Valine & 108 & 4.85 & 102 & 7.02 & 103 & 1.84 & 101 & 2.37 & 95.6 & 1.96 & 105 & 5.82 & 98.8 & 2.76 & 101 & 3.38 \\
\hline Threonine & 97.7 & 2.22 & 97.3 & 5.81 & 104 & 2.02 & 106 & 8.68 & 92.1 & 2.18 & 93.5 & 6.10 & 98.8 & 3.42 & 95.8 & 3.03 \\
\hline Phenylethylamine & 105 & 1.81 & 102 & 7.79 & 103 & 3.31 & 102 & 7.18 & 99.9 & 4.26 & 101 & 6.78 & 103 & 2.66 & 106 & 6.47 \\
\hline Taurine & 116 & 3.47 & 106 & 8.51 & 98.3 & 2.94 & 107 & 1.97 & 102 & 1.30 & 92.1 & 1.34 & 100 & 5.19 & 94.1 & 1.26 \\
\hline Putrescine & 97.1 & 2.13 & 97.2 & 5.39 & 110 & 1.37 & 107 & 3.60 & 110 & 3.59 & 104 & 4.30 & 110 & 1.00 & 99.3 & 7.10 \\
\hline cis-Hydroxyproline & 98.8 & 5.92 & 97.1 & 7.49 & 99.2 & 3.91 & 106 & 6.08 & 105 & 1.37 & 96.5 & 6.37 & 101 & 1.46 & 106 & 4.54 \\
\hline $\begin{array}{c}\text { trans- } \\
\text { Hydroxyproline }\end{array}$ & 97.1 & 1.39 & 96.5 & 8.59 & 111 & 7.04 & 96.7 & 2.54 & 101 & 4.35 & 100 & 5.03 & 100 & 2.62 & 102 & 3.41 \\
\hline Leucine & 107 & 3.21 & 103 & 6.29 & 96.3 & 2.01 & 105 & 4.54 & 92.9 & 2.05 & 103 & 3.81 & 93.0 & 0.699 & 97.8 & 5.98 \\
\hline Isoleucine & 98.1 & 3.41 & 102 & 5.53 & 102 & 2.61 & 98.1 & 5.06 & 102 & 4.32 & 99.1 & 2.73 & 98.8 & 1.14 & 106 & 2.59 \\
\hline Asparagine & 110 & 1.78 & 108 & 3.94 & 102 & 3.72 & 110 & 5.09 & 95.0 & 1.32 & 108 & 3.40 & 102 & 4.29 & 101 & 2.76 \\
\hline Aspartic acid & 105 & 4.95 & 105 & 4.35 & 102 & 1.13 & 108 & 4.20 & 100 & 4.50 & 103 & 5.07 & 106 & 3.40 & 100 & 2.93 \\
\hline Glutamine & 108 & 7.39 & 99.8 & 5.74 & 114 & 2.19 & 104 & 4.20 & 105 & 7.54 & 102 & 5.03 & 106 & 4.91 & 95.8 & 3.68 \\
\hline Glutamic acid & 103 & 6.59 & 99.7 & 4.83 & 115 & 1.24 & 103 & 9.08 & 102 & 2.67 & 104 & 7.29 & 102 & 5.55 & 107 & 6.40 \\
\hline Methionine & 105 & 4.65 & 98.7 & 6.14 & 109 & 1.14 & 108 & 5.33 & 102 & 2.14 & 103 & 3.01 & 102 & 5.01 & 102 & 6.29 \\
\hline Dopamine & 93.6 & 6.62 & 97.5 & 7.51 & 100 & 4.26 & 100 & 5.98 & 98.5 & 2.23 & 98.9 & 4.88 & 100 & 3.53 & 102 & 3.17 \\
\hline Histidine & 113 & 4.98 & 104 & 7.64 & 112 & 0.257 & 110 & 3.94 & 101 & 2.41 & 104 & 3.31 & 94.8 & 2.32 & 102 & 4.43 \\
\hline
\end{tabular}




\begin{tabular}{|c|c|c|c|c|c|c|c|c|c|c|c|c|c|c|c|c|}
\hline $\begin{array}{l}\text { alpha-aminoadipic } \\
\text { acid }\end{array}$ & 107 & 6.34 & 110 & 7.45 & 108 & 4.62 & 107 & 7.17 & 114 & 2.28 & 101 & 7.01 & 97.2 & 0.557 & 105 & 1.33 \\
\hline Phenylalanine & 101 & 2.54 & 95.8 & 4.92 & 105 & 1.43 & 107 & 3.93 & 99.0 & 4.11 & 99.3 & 2.87 & 104 & 3.23 & 98.5 & 1.81 \\
\hline $\begin{array}{l}\text { Methionine- } \\
\text { Sulfoxide }\end{array}$ & 95.4 & 0.378 & 95.2 & 4.49 & 101 & 1.98 & 109 & 7.53 & 103 & 3.51 & 106 & 7.71 & 103 & 5.09 & 101 & 4.59 \\
\hline Arginine & 115 & 7.47 & 112 & 8.64 & 105 & 2.61 & 106 & 1.92 & 104 & 2.41 & 101 & 2.37 & 97.5 & 1.70 & 96.9 & 1.64 \\
\hline Acetyl-ornithine & 104 & 1.66 & 102 & 2.69 & 113 & 0.798 & 109 & 3.24 & 114 & 2.89 & 112 & 4.03 & 116 & 2.25 & 107 & 6.62 \\
\hline Citrulline & 99.6 & 3.41 & 97.5 & 8.53 & 106 & 4.11 & 104 & 6.30 & 96.1 & 2.10 & 104 & 6.91 & 94.5 & 2.70 & 97.2 & 2.94 \\
\hline Serotonin & 111 & 2.33 & 103 & 8.48 & 104 & 1.62 & 102 & 7.14 & 101 & 2.42 & 100 & 1.65 & 101 & 1.03 & 98.5 & 3.48 \\
\hline Tyrosine & 95.9 & 3.81 & 98.7 & 6.59 & 108 & 3.03 & 113 & 5.56 & 91.7 & 3.07 & 99.4 & 7.99 & 101 & 4.10 & 92.9 & 7.93 \\
\hline DOPA & 93.8 & 6.51 & 93.8 & 6.38 & 108 & 0.835 & 107 & 4.30 & 103 & 3.54 & 101 & 2.20 & 103 & 3.81 & 96.5 & 2.76 \\
\hline $\begin{array}{c}\text { Asymmetric } \\
\text { dimethylarginine }\end{array}$ & 97.9 & 7.75 & 95.7 & 6.93 & 104 & 1.58 & 102 & 3.71 & 110 & 2.42 & 101 & 7.54 & 104 & 5.09 & 97.7 & 6.76 \\
\hline $\begin{array}{c}\text { Total } \\
\text { dimethylarginine }\end{array}$ & 96.3 & 8.34 & 96.7 & 4.98 & 97.5 & 2.22 & 111 & 6.85 & 94.6 & 3.49 & 103 & 1.40 & 95.6 & 2.36 & 93.9 & 2.52 \\
\hline Tryptophan & 99.7 & 0.261 & 99.8 & 3.53 & 107 & 2.50 & 104 & 1.77 & 101 & 0.711 & 104 & 3.41 & 101 & 0.991 & 99.2 & 5.00 \\
\hline Kynurenine & 102 & 1.28 & 102 & 6.86 & 100 & 2.56 & 106 & 2.94 & 90.0 & 3.76 & 92.3 & 5.19 & 101 & 4.53 & 90.6 & 6.17 \\
\hline Carnosine & 106 & 2.68 & 104 & 4.31 & 111 & 3.70 & 101 & 4.16 & 90.3 & 4.28 & 100 & 3.96 & 90.1 & 4.36 & 96.1 & 6.40 \\
\hline Nitro-Tyrosine & 93.6 & 7.02 & 96.2 & 5.42 & 103 & 6.95 & 99.5 & 5.80 & 108 & 1.46 & 97.6 & 3.51 & 105 & 3.22 & 91.6 & 3.28 \\
\hline Ornithine & 106 & 1.27 & 108 & 4.99 & 115 & 2.53 & 111 & 6.77 & 98.5 & 5.76 & 99.1 & 5.92 & 100 & 8.14 & 96.6 & 4.75 \\
\hline Lysine & 100 & 3.96 & 102 & 5.23 & 99.0 & 2.19 & 108 & 5.08 & 92.8 & 1.87 & 96.4 & 5.03 & 101 & 1.64 & 105 & 4.95 \\
\hline Spermidine & 105 & 5.14 & 102 & 2.81 & 106 & 2.51 & 103 & 5.77 & 106 & 2.31 & 97.3 & 3.81 & 103 & 0.928 & 98.9 & 3.96 \\
\hline Spermine & 104 & 3.95 & 106 & 4.56 & 95.8 & 4.73 & 107 & 5.44 & 107 & 2.57 & 100 & 4.75 & 108 & 3.20 & 102 & 4.60 \\
\hline Sarcosine & 103 & 5.74 & 98.9 & 4.51 & 95.4 & 0.545 & 99.2 & 5.99 & 97.9 & 4.10 & 98.3 & 5.44 & 101 & 3.23 & 99.1 & 1.78 \\
\hline Diacetylspermine & 102 & 5.73 & 103 & 5.71 & 98.3 & 7.27 & 105 & 3.19 & 98.5 & 5.03 & 99.4 & 4.17 & 100 & 1.65 & 103 & 5.26 \\
\hline Tyramine & 90.1 & 5.11 & 97.7 & 9.27 & 102 & 2.32 & 102 & 2.79 & 94.0 & 3.78 & 102 & 3.65 & 102 & 4.39 & 97.4 & 4.04 \\
\hline Creatine & 107 & 3.49 & 101 & 5.75 & 108 & 3.04 & 106 & 2.94 & 106 & 1.59 & 104 & 3.46 & 105 & 3.63 & 99.4 & 2.63 \\
\hline Phosphocreatine & 95.4 & 5.61 & 94.1 & 4.17 & 96.5 & 7.85 & 98.2 & 8.81 & 91.0 & 4.44 & 96 & 5.57 & 89.1 & 2.49 & 96.7 & 3.33 \\
\hline Betaine & 103 & 0.609 & 106 & 4.66 & 96.4 & 1.28 & 103 & 4.69 & 99.6 & 2.20 & 102 & 3.64 & 105 & 3.28 & 105 & 4.45 \\
\hline Choline & 99.5 & 9.15 & 101 & 4.67 & 101 & 5.59 & 97.8 & 2.10 & 107 & 5.92 & 104 & 5.26 & 102 & 0.769 & 105 & 3.98 \\
\hline TMAO & 99.9 & 3.25 & 98.1 & 4.02 & 116 & 0.647 & 104 & 6.17 & 102 & 1.23 & 100 & 4.96 & 93.4 & 1.16 & 103 & 2.83 \\
\hline Methylhistidine & 95.2 & 0.758 & 99.4 & 3.64 & 112 & 0.734 & 113 & 7.48 & 92.7 & 0.436 & 107 & 4.08 & 96.4 & 3.00 & 101 & 3.29 \\
\hline Lactic acid & 93.4 & 1.98 & 104 & 7.32 & 105 & 0.232 & 95.7 & 5.46 & 105 & 1.53 & 103 & 2.19 & 107 & 2.54 & 98.3 & 1.87 \\
\hline
\end{tabular}




\begin{tabular}{|c|c|c|c|c|c|c|c|c|c|c|c|c|c|c|c|c|}
\hline $\begin{array}{c}\text { Beta- } \\
\text { Hydroxybutyric acid }\end{array}$ & 97.7 & 4.65 & 103 & 5.31 & 105 & 1.53 & 93.7 & 1.57 & 104 & 0.664 & 99.0 & 4.44 & 106 & 4.04 & 100 & 3.17 \\
\hline $\begin{array}{l}\text { Alpha-Ketoglutaric } \\
\text { acid }\end{array}$ & 97.2 & 0.381 & 99.1 & 4.29 & 113 & 2.29 & 93.6 & 1.01 & 112 & 1.86 & 106 & 4.19 & 106 & 4.11 & 99.7 & 4.22 \\
\hline Citric acid & 96.8 & 0.826 & 97.8 & 6.49 & 110 & 0.483 & 93.2 & 1.61 & 109 & 0.917 & 103 & 2.84 & 109 & 3.99 & 96.5 & 3.83 \\
\hline Butyric acid & 98.1 & 4.11 & 99.2 & 3.51 & 101 & 2.95 & 101 & 7.40 & 102 & 2.26 & 100 & 2.90 & 101 & 2.50 & 99.0 & 2.37 \\
\hline Propionic acid & 95.5 & 2.69 & 101 & 5.27 & 108 & 2.95 & 97.3 & 5.40 & 94.0 & 2.48 & 96.9 & 0.987 & 112 & 0.927 & 95.0 & 4.65 \\
\hline HPHPA & 91.4 & 1.14 & 99.2 & 8.17 & 111 & 1.02 & 93.3 & 2.24 & 111 & 1.97 & 103 & 0.783 & 109 & 2.91 & 96.6 & 2.66 \\
\hline $\begin{array}{c}\text { para- } \\
\text { Hydroxyhippuric } \\
\text { acid }\end{array}$ & 89.4 & 3.24 & 91.1 & 3.41 & 99.5 & 2.82 & 93.8 & 6.20 & 94.7 & 0.880 & 98.2 & 4.70 & 98.6 & 0.537 & 93.8 & 3.46 \\
\hline Succinic acid & 100 & 2.03 & 101 & 3.82 & 108 & 1.75 & 90.6 & 5.20 & 108 & 0.284 & 101 & 2.37 & 108 & 3.34 & 97.7 & 3.32 \\
\hline Fumaric acid & 101 & 1.21 & 101 & 5.12 & 95.3 & 3.81 & 93.1 & 0.768 & 95.2 & 4.34 & 94.0 & 5.04 & 101 & 4.95 & 95.7 & 3.90 \\
\hline Pyruvic acid & 97.8 & 1.73 & 103 & 6.14 & 110 & 0.730 & 93.7 & 3.67 & 107 & 2.07 & 107 & 4.01 & 105 & 4.20 & 101 & 6.17 \\
\hline Isobutyric acid & 96.9 & 2.76 & 96.1 & 3.61 & 100 & 2.56 & 94.7 & 6.32 & 105 & 6.17 & 106 & 3.45 & 96.0 & 0.417 & 100 & 2.29 \\
\hline Hippuric acid & 96.7 & 0.382 & 102 & 6.65 & 107 & 3.40 & 92.7 & 4.91 & 106 & 3.27 & 100 & 5.99 & 98.7 & 1.28 & 97.8 & 2.37 \\
\hline Methylmalonic acid & 95.7 & 1.74 & 102 & 7.68 & 101 & 1.10 & 92.2 & 2.97 & 99.2 & 1.40 & 100 & 1.88 & 102 & 3.63 & 97.8 & 5.36 \\
\hline Homovanillic acid & 102 & 2.89 & 106 & 2.99 & 104 & 4.54 & 100 & 3.71 & 102 & 4.33 & 97.8 & 5.69 & 100 & 4.23 & 94.9 & 2.36 \\
\hline Indole acetic acid & 94.7 & 3.28 & 99.1 & 3.62 & 107 & 2.39 & 92.4 & 2.06 & 105 & 0.884 & 98.6 & 4.40 & 104 & 2.25 & 94.6 & 3.34 \\
\hline Uric acid & 94.6 & 3.84 & 103 & 6.63 & 105 & 1.54 & 102 & 3.21 & 103 & 0.850 & 97.7 & 2.99 & 108 & 4.04 & 95.4 & 2.74 \\
\hline
\end{tabular}


Table S5. Recovery Performance in Spiked Pooled Human Urine

\begin{tabular}{|c|c|c|c|}
\hline \multirow{2}{*}{ Analyte } & \multicolumn{3}{|c|}{ Recovery (\%) } \\
\hline & Low & Mid & High \\
\hline Creatinine & 97.5 & 97.3 & 101 \\
\hline Glycine & 107 & 100 & 99.3 \\
\hline Alanine & 106 & 101 & 101 \\
\hline Serine & 104 & 99 & 101 \\
\hline Histamine & 99.3 & 100 & 102 \\
\hline Proline & 101 & 103 & 99.2 \\
\hline Valine & 112 & 100 & 102 \\
\hline Threonine & 101 & 95 & 104 \\
\hline Phenylethylamine & 112 & 107 & 106 \\
\hline Taurine & 104 & 102 & 103 \\
\hline Putrescine & 101 & 101 & 100 \\
\hline cis-Hydroxyproline & 98.5 & 102 & 102 \\
\hline trans-Hydroxyproline & 98.7 & 100 & 103 \\
\hline Leucine & 102 & 92 & 100 \\
\hline Isoleucine & 94.0 & 97.7 & 92.6 \\
\hline Asparagine & 101 & 87.3 & 98.2 \\
\hline Aspartic acid & 103 & 98.1 & 96.6 \\
\hline Glutamine & 104 & 100 & 102 \\
\hline Glutamic acid & 98.1 & 98.5 & 97.9 \\
\hline Methionine & 103 & 100 & 103 \\
\hline Dopamine & 103 & 101 & 103 \\
\hline Histidine & 104 & 90.8 & 92.5 \\
\hline alpha-aminoadipic acid & 93.4 & 92.8 & 91.6 \\
\hline Phenylalanine & 107 & 103 & 104 \\
\hline Methionine-Sulfoxide & 102 & 97.2 & 97.7 \\
\hline Arginine & 111 & 110 & 102 \\
\hline Acetyl-ornithine & 96.3 & 95.8 & 94.5 \\
\hline Citrulline & 105 & 103 & 100 \\
\hline Serotonin & 101 & 103 & 104 \\
\hline Tyrosine & 94.2 & 95.4 & 97.1 \\
\hline DOPA & 104 & 97.3 & 101 \\
\hline Asymmetric dimethylarginine & 114 & 100 & 99.2 \\
\hline Total dimethylarginine & 104 & 100 & 101 \\
\hline Tryptophan & 107 & 103 & 109 \\
\hline Kynurenine & 96.3 & 93.7 & 102 \\
\hline Carnosine & 99.0 & 106 & 109 \\
\hline Nitro-Tyrosine & 103 & 101 & 98.5 \\
\hline
\end{tabular}




\begin{tabular}{|c|c|c|c|}
\hline Ornithine & 102 & 95.9 & 99.2 \\
\hline Lysine & 93.2 & 103 & 98.5 \\
\hline Spermidine & 92.5 & 98.5 & 98.8 \\
\hline Spermine & 94.4 & 107 & 100 \\
\hline Sarcosine & 103 & 104 & 97.2 \\
\hline Diacetylspermine & 100 & 102 & 102 \\
\hline Tyramine & 96.4 & 102 & 97.9 \\
\hline Creatine & 97.5 & 102 & 98.0 \\
\hline Phosphocreatine & 93.3 & 100 & 98.2 \\
\hline Betaine & 108 & 106 & 107 \\
\hline Choline & 99.4 & 95.9 & 94.5 \\
\hline TMAO & 113 & 103 & 102 \\
\hline Methylhistidine & 98.2 & 101 & 97.5 \\
\hline Lactic acid & 102 & 96.2 & 109 \\
\hline Beta-Hydroxybutyric acid & 104 & 94.2 & 107 \\
\hline Alpha-Ketoglutaric acid & 108 & 98.1 & 102 \\
\hline Citric acid & 112 & 94.5 & 114 \\
\hline Butyric acid & 103 & 97.5 & 101 \\
\hline Propionic acid & 105 & 108 & 102 \\
\hline HPHPA & 96.4 & 97.5 & 104 \\
\hline para-Hydroxyhippuric acid & 100 & 96.0 & 110 \\
\hline Succinic acid & 103 & 91.5 & 100 \\
\hline Fumaric acid & 104 & 90.4 & 97.4 \\
\hline Pyruvic acid & 115 & 103 & 109 \\
\hline Isobutyric acid & 90.8 & 96.1 & 93.1 \\
\hline Hippuric acid & 109 & 106 & 93.4 \\
\hline Methylmalonic acid & 96.7 & 93.9 & 105 \\
\hline Homovanillic acid & 107 & 102 & 109 \\
\hline Indole acetic acid & 101 & 92.8 & 103 \\
\hline Uric acid & 110 & 102 & 104 \\
\hline
\end{tabular}


Table S6. Validation Performance of Analytes Analyzed via DFI-MS/MS

\begin{tabular}{|c|c|c|c|c|c|c|c|c|}
\hline Analyte & Full Name & $\begin{array}{l}\text { LOD } \\
(\mu \mathrm{M})\end{array}$ & $\begin{array}{c}\text { Accuracy } \\
\text { (\%) }\end{array}$ & $\begin{array}{l}\text { CV } \\
(\%)\end{array}$ & $\begin{array}{c}\text { Recovery } \\
\text { (\%) }\end{array}$ & $\begin{array}{c}3 X \\
\text { Dilution } \\
\text { Recovery } \\
(\%)\end{array}$ & $\begin{array}{c}5 X \\
\text { Dilution } \\
\text { Recovery } \\
(\%)^{*}\end{array}$ & $\begin{array}{c}10 \mathrm{X} \\
\text { Dilution } \\
\text { Recovery } \\
(\%)^{*}\end{array}$ \\
\hline LysoPC a C14:0 & LysoPhosphatidylcholine acyl C14:0 & 1.9 & I & I & I & 98.5 & / & 1 \\
\hline LysoPC a C16:1 & LysoPhosphatidylcholine acyl C16:1 & 0.209 & I & I & I & 97.9 & I & I \\
\hline LysoPC a C16:0 & LysoPhosphatidylcholine acyl C16:0 & 0.753 & / & / & / & 97.4 & / & / \\
\hline LysoPC a C17:0 & LysoPhosphatidylcholine acyl C17:0 & 0.275 & / & / & / & 92.8 & / & / \\
\hline LysoPC a C18:2 & LysoPhosphatidylcholine acyl C18:2 & 0.415 & I & I & I & 89.1 & I & I \\
\hline LysoPC a C18:1 & LysoPhosphatidylcholine acyl C18:1 & 0.107 & 115 & 6.65 & 108 & 109 & I & I \\
\hline LysoPC a C18:0 & LysoPhosphatidylcholine acyl C18:0 & 0.344 & / & I & I & 90.9 & I & I \\
\hline LysoPC a C20:4 & LysoPhosphatidylcholine acyl C20:4 & 0.243 & / & / & / & 93.2 & / & / \\
\hline LysoPC a C20:3 & LysoPhosphatidylcholine acyl C20:3 & 0.331 & I & I & I & 91.1 & I & I \\
\hline LysoPC a C24:0 & LysoPhosphatidylcholine acyl C24:0 & 0.143 & I & I & I & 105 & I & I \\
\hline LysoPC a C26:1 & LysoPhosphatidylcholine acyl C26:1 & 0.0327 & / & / & / & 107 & / & / \\
\hline LysoPC a C26:0 & LysoPhosphatidylcholine acyl C26:0 & 0.0444 & I & I & I & 109 & I & I \\
\hline LysoPC a C28:1 & LysoPhosphatidylcholine acyl C28:1 & 0.0183 & I & I & I & 91.9 & I & I \\
\hline LysoPC a C28:0 & LysoPhosphatidylcholine acyl C28:0 & 0.106 & I & I & I & 89.1 & I & I \\
\hline $\mathrm{SM}(\mathrm{OH}) \mathrm{C} 14: 1$ & Hydroxysphingomyelin C14:1 & 0.0186 & / & / & / & 92.2 & / & / \\
\hline SM C16:1 & Sphingomyelin C16:1 & 0.0117 & I & / & I & 94.8 & I & I \\
\hline SM C16:0 & Sphingomyelin C16:0 & 0.0423 & I & I & I & 112 & I & I \\
\hline $\mathrm{SM}(\mathrm{OH}) \mathrm{C} 16: 1$ & Hydroxysphingomyelin C16:1 & 0.0139 & / & / & / & 87.9 & / & / \\
\hline SM C18:1 & Sphingomyelin C18:1 & 0.00969 & / & / & / & 87.1 & / & / \\
\hline PC aa C32:2 & Phosphatidylcholine diacyl C32:2 & 0.0613 & I & I & I & 106 & I & I \\
\hline SM C18:0 & Sphingomyelin C18:0 & 0.0914 & 86.5 & 8.86 & 87.5 & 94.1 & I & I \\
\hline SM C20:2 & Sphingomyelin C20:2 & 0.145 & I & I & I & 97.2 & I & I \\
\hline PC ae C36:0 & Phosphatidylcholine acyl-alkyl C36:0 & 0.0252 & / & / & / & 85.7 & / & / \\
\hline PC aa C36:6 & Phosphatidylcholine diacyl C36:6 & 0.0159 & I & I & I & 105 & I & I \\
\hline PC aa C36:0 & Phosphatidylcholine diacyl C36:0 & 0.0829 & 114 & 8.05 & 93.9 & 99.9 & / & I \\
\hline
\end{tabular}




\begin{tabular}{|c|c|c|c|c|c|c|c|c|}
\hline $\mathrm{SM}(\mathrm{OH}) \mathrm{C} 22: 2$ & Hydroxysphingomyelin C22:2 & 0.0183 & / & / & / & 87.8 & / & / \\
\hline $\mathrm{SM}(\mathrm{OH}) \mathrm{C} 22: 1$ & Hydroxysphingomyelin C22:1 & 0.0231 & I & / & l & 87.8 & I & I \\
\hline PC aa C38:6 & Phosphatidylcholine diacyl C38:6 & 0.0249 & / & / & / & 114 & / & / \\
\hline Pc aa C38:0 & Phosphatidylcholine diacyl C38:0 & 0.602 & / & / & / & 97.5 & / & / \\
\hline Pc ae C40:6 & Phosphatidylcholine acyl-alkyl C40:6 & 0.0849 & I & / & / & 89.9 & / & / \\
\hline $\mathrm{SM}(\mathrm{OH}) \mathrm{C} 24: 1$ & Hydroxysphingomyelin C24:1 & 0.0243 & I & I & I & 85.2 & I & I \\
\hline PC aa C40:6 & Phosphatidylcholine diacyl C40:6 & 0.0688 & 1 & I & I & 86.9 & I & I \\
\hline PC aa C40:2 & Phosphatidylcholine diacyl C40:2 & 0.0107 & / & / & / & 107 & / & / \\
\hline PC aa C40:1 & Phosphatidylcholine diacyl C40:1 & 0.147 & I & / & I & 88.5 & I & I \\
\hline $\mathrm{CO}$ & Carnitine & 2.12 & 92.6 & 7.85 & 102 & 97.2 & 96.9 & 94.7 \\
\hline $\mathrm{C} 2$ & Acetylcarnitine & 0.135 & 116 & 9.72 & 99.9 & 90.9 & 95.2 & 92.9 \\
\hline C3:1 & Propenoylcarnitine & 0.0375 & I & I & I & 105 & I & I \\
\hline $\mathrm{C3}$ & Propionylcarnitine & 0.0841 & 90.9 & 4.41 & 90.6 & 104 & 108 & 101 \\
\hline $\mathrm{C} 4: 1$ & Butenylcarnitine & 0.0301 & I & I & I & 104 & 113 & 105 \\
\hline $\mathrm{C} 4$ & Butyrylcarnitine & 0.0491 & 113 & 11.6 & 118 & 96.5 & 95.7 & 93.6 \\
\hline $\mathrm{C} 3 \mathrm{OH}$ & Hydroxypropionylcarnitine & 0.0348 & 1 & I & 1 & 111 & 108 & 112 \\
\hline $\mathrm{C} 5: 1$ & Tiglylcarnitine & 0.0309 & / & / & / & 90.2 & 94.6 & 96.7 \\
\hline $\mathrm{C5}$ & Valerylcarnitine & 0.0253 & 111 & 10.2 & 108 & 89.3 & 89.2 & 94.1 \\
\hline $\mathrm{C} 4 \mathrm{OH}$ & Hydroxybutyrylcarnitine & 0.0308 & I & I & I & 106 & 109 & 108 \\
\hline C6:1 & Hexenoylcarnitine & 0.0245 & I & I & I & 103 & 114 & 108 \\
\hline $\mathrm{C} 6$ & Hexanoylcarnitine & 0.113 & 95.5 & 5.67 & 87.2 & 95.2 & 91.4 & 91.3 \\
\hline $\mathrm{C} 5 \mathrm{OH}$ & Hydroxyvalerylcarnitine & 0.0330 & 1 & I & I & 102 & 97.8 & 91.5 \\
\hline $\mathrm{C} 5: 1 \mathrm{DC}$ & Glutaconylcarnitine & 0.0270 & I & I & I & 88.3 & 84.4 & 88.9 \\
\hline C5DC & Glutarylcarnitine & 0.0181 & I & I & 1 & 99.9 & 93.1 & 89.6 \\
\hline $\mathrm{C} 8$ & Octanoylcarnitine & 0.339 & 95.1 & 4.79 & 113 & 102 & 102 & 105 \\
\hline C5MDC & Methylglutarylcarnitine & 0.0227 & I & I & I & 102 & 98.1 & 96.5 \\
\hline C9 & Nonaylcarnitine & 0.0193 & I & I & 1 & 85.5 & 89.2 & 90.3 \\
\hline C7DC & Pimelylcarnitine & 0.0208 & 1 & 1 & 1 & 94.2 & 88.6 & 89.2 \\
\hline C10:2 & Decadienylcarnitine & 0.0438 & I & I & I & 96.6 & 90.2 & 91.6 \\
\hline C10:1 & Decenoylcarnitine & 0.136 & I & I & I & 94.3 & 87.1 & 88.5 \\
\hline C10 & Decanoylcarnitine & 0.0598 & 96.7 & 8.62 & 94.9 & 91.2 & 91.4 & 88.5 \\
\hline
\end{tabular}




\begin{tabular}{|c|c|c|c|c|c|c|c|c|}
\hline $\mathrm{C} 12: 1$ & Dodecenoylcarnitine & 0.0825 & $/$ & $/$ & $/$ & 102 & 108 & 108 \\
\hline $\mathrm{C} 12$ & Dodecanoylcarnitine & 0.0439 & 117 & 7.67 & 119 & 115 & 117 & 117 \\
\hline $\mathrm{C} 14: 2$ & Tetradecadienylcarnitine & 0.0126 & $/$ & $/$ & $/$ & 104 & $/$ & $/$ \\
\hline $\mathrm{C} 14: 1$ & Tetradecenoylcarnitine & 0.0169 & $/$ & $/$ & $/$ & 113 & $/$ & $/$ \\
\hline $\mathrm{C} 14$ & Tetradecanoylcarnitine & 0.0344 & 104 & 8.25 & 105 & 110 & $/$ & $/$ \\
\hline $\mathrm{C} 12 \mathrm{DC}$ & Dodecanedioylcarnitine & 0.0206 & $/$ & $/$ & $/$ & 112 & $/$ & $/$ \\
\hline $\mathrm{C} 14: 2 \mathrm{OH}$ & Hydroxytetradecadienylcarnitine & 0.0184 & $/$ & $/$ & $/$ & 101 & $/$ & $/$ \\
\hline $\mathrm{C} 14: 1 \mathrm{OH}$ & Hydroxytetradecenoylcarnitine & 0.0126 & $/$ & $/$ & $/$ & 106 & $/$ & $/$ \\
\hline $\mathrm{C} 16: 2$ & Hexadecadienylcarnitine & 0.0189 & $/$ & $/$ & $/$ & 105 & $/$ & $/$ \\
\hline $\mathrm{C} 16: 1$ & Hexadecenoylcarnitine & 0.0242 & $/$ & $/$ & $/$ & 112 & $/$ & $/$ \\
\hline $\mathrm{C} 16$ & Hexadecanoylcarnitine & 0.0264 & 95.2 & 5.13 & 103 & 109 & $/$ & $/$ \\
\hline $\mathrm{C} 16: 2 \mathrm{OH}$ & Hydroxyhexadecadienylcarnitine & 0.0136 & $/$ & $/$ & $/$ & 105 & $/$ & $/$ \\
\hline $\mathrm{C} 16: 1 \mathrm{H}$ & Hydroxyhexadecenoylcarnitine & 0.0123 & $/$ & $/$ & $/$ & 105 & $/$ & $/$ \\
\hline $\mathrm{C} 16 \mathrm{OH}$ & Hydroxyhexadecanoylcarnitine & 0.0867 & $/$ & $/$ & $/$ & 106 & $/$ & $/$ \\
\hline $\mathrm{C} 18: 2$ & Octadecadienylcarnitine & 0.0622 & $/$ & $/$ & $/$ & 103 & $/$ & $/$ \\
\hline $\mathrm{C} 18: 1$ & Octadecenoylcarnitine & 0.0167 & $/$ & $/$ & $/$ & 104 & $/$ & $/$ \\
\hline $\mathrm{C} 18$ & Octadecanoylcarnitine & 0.0241 & 104 & 9.25 & 98.6 & 98.1 & $/$ & $/$ \\
\hline $\mathrm{C} 18: 1 \mathrm{OH}$ & Hydroxyoctadecenoylcarnitine & 0.0125 & $/$ & $/$ & $/$ & 105 & $/$ & $/$ \\
\hline $\mathrm{Gluc0se}$ & Glucose & 18.5 & 96.3 & 4.90 & 96.4 & 95.8 & 93.7 & 93.1 \\
\hline
\end{tabular}

*Recoveries of $5 \mathrm{X}$ and 10X dilutions were not available for some analytes that were not detected after dilutions. 


\section{Table S7. Method Comparison to Other Urinary Assays}

\begin{tabular}{|c|c|c|c|c|c|c|c|c|}
\hline \multirow[b]{2}{*}{ Method } & \multicolumn{3}{|c|}{ No. of quantified metabolites } & \multirow{2}{*}{$\begin{array}{l}\text { Assay } \\
\text { Type }\end{array}$} & \multirow{2}{*}{$\begin{array}{c}\text { Sample } \\
\text { analysis } \\
\text { time (min) }\end{array}$} & \multirow{2}{*}{$\begin{array}{l}\text { No. of data } \\
\text { acquisitions }\end{array}$} & \multirow{2}{*}{$\begin{array}{l}\text { Validation } \\
\text { on urine } \\
\text { samples }\end{array}$} & \multirow[b]{2}{*}{ Compound classes } \\
\hline & $\begin{array}{l}\text { Full (No. of } \\
\text { ISTDs used) }\end{array}$ & Semi & Relative & & & & & \\
\hline This Assay & $67(50)$ & 75 & 0 & LC-MS & 33.5 & 4 & Yes & $\begin{array}{c}\text { amino acid, biogenic amine, organic acids, sugars, } \\
\text { acylcarnitines, lipids }\end{array}$ \\
\hline Biocrates $^{\circledR}$ p180 kit ${ }^{35}$ & $41(27)$ & 62 & 0 & LC-MS & 15.5 & 3 & No & $\begin{array}{c}\text { amino acid, biogenic amine, sugars, acylcarnitines, } \\
\text { lipids }\end{array}$ \\
\hline Assay 119 & 0 & 93 & 0 & LC-MS & 12 & 1 & No & amino acids, biogenic amines \\
\hline Assay 226 & 0 & 108 & 0 & LC-MS & 40 & 1 & No & amino acids, organic acids, sugars, vitamins \\
\hline Assay 329 & $57(16)$ & 0 & 0 & LC-MS & 15 & 1 & Yes & bile acids \\
\hline Assay $4^{30}$ & 0 & 0 & 112 & LC-MS & 51 & 3 & No & amino acids, organic acids, sugars, nucleotides \\
\hline Assay $5^{31}$ & 0 & 108 & 0 & LC-MS & 40 & 1 & No & amino acids, organic acids, sugars, vitamins \\
\hline Assay $6^{17}$ & $85(2)$ & 0 & 0 & GC-MS & 92 & 2 & Yes & amino acids, organic acids, sugars \\
\hline Assay 724 & $20(2)$ & 0 & 82 & GC-MS & $>50$ & 1 & No & organic acids \\
\hline Assay 825 & $75(0)$ & 0 & 0 & GC-MS & 30 & 1 & N/A & amino acid, organic acid, sugar, nucleic acids \\
\hline Assay 927 & $95(0)$ & 0 & 0 & GC-MS & 75 & 1 & $\mathrm{~N} / \mathrm{A}$ & amino acids, organic acids, sugars \\
\hline Assay $10^{28}$ & $67(67)$ & 0 & 0 & GC-MS & 15.5 & 1 & Yes & amino acids, organic acids \\
\hline Assay $11^{33}$ & $112(2)$ & 0 & 0 & GC-MS & 38 & 1 & No & amino acids, organic acids \\
\hline Assay $12^{34}$ & $69(0)$ & 0 & 0 & GC-MS & 88 & 1 & $\mathrm{~N} / \mathrm{A}$ & organic acids \\
\hline Assay $13^{32}$ & 0 & 53 & 0 & CE-MS & 30 & 1 & No & $\begin{array}{c}\text { amino acids, biogenic amines, nucleic acids, small } \\
\text { peptides }\end{array}$ \\
\hline
\end{tabular}

